\title{
The Importance of Teaching Risk Assessment and Safety-Netting Skills in Primary Care
}

\section{Paul Silverston MBChB ${ }^{1-3 *}$}

${ }^{1}$ Director of Primary Care Education and Hon. Visiting Senior Lecturer, The Postgraduate Medical Institute, Anglia Ruskin University, Chelmsford, England

${ }^{2}$ Senior Lecturer in Clinical Assessment Teaching, Department of Primary Care and Public Health, Anglia Ruskin University, Cambridge, England

${ }^{3}$ Visiting Senior Fellow in Medical Education, University Campus Suffolk, Ipswich, England

\begin{abstract}
Medical students and junior doctors learn how to recognise patterns of illness from textbooks and disease-specific lectures that tend to present illnesses in their established form, when the symptom history, clinical findings and investigations are all positive for that disease. This is further reinforced by seeing patients in hospital clinics and on the wards who have established disease and in whom the diagnosis has often already been made. In primary care, patients often present much earlier in their illness with symptoms and signs that are not disease-specific and yet they may still have a serious illness which has yet to manifest itself through the presence of positive findings in the symptom history, or clinical examination. Medical students and junior doctors on attachments in primary care may not be familiar with the risks inherent in assessing patients during the early stages of a serious illness that they would otherwise be familiar with in its established form and also of the limitations of performing a single point in time clinical assessment during the early stages of an evolving illness. This paper discusses the role that medical educators based within the primary care setting have in teaching medical students and junior doctors about the need to perform a risk assessment when patients present during the early stages of an illness and the importance of safety-netting these patients, if they are to be managed safely.
\end{abstract}

Keywords: Model of illness; Consultation skills; Clinical assessment; Risk assessment; Patient safety; Primary care

\section{Introduction}

Medical students and junior doctors usually begin their medical education by observing or conducting consultations within the hospital setting before doing so in primary care. The patterns of illness that the medical student and junior doctor learn from textbooks and disease specific lectures tend to be those of established disease and not those of early and evolving disease, in which the symptoms and signs tend to be non-specific and non-diagnostic [1]. This is further reinforced by seeing patients with established disease in the clinic or ward setting, where there are usually positive and confirmatory findings in the patient's history, clinical examination and investigations. This means that the clinical assessment is being performed backwards from the diagnosis that has been made towards the symptoms that the patient initially presented with, which contrasts with the way in which patients present in primary care. An attachment in primary care affords the medical student or junior doctor an excellent opportunity not only to learn about the dynamic nature of illness and the patterns of illness associated with early and evolving disease but also how to work forwards from the symptoms being presented by the patient using a symptom-based, patient safety focused clinical assessment.

\section{The Nature of Illness}

The relationship between illness and time is such that patients with the same disease will present with differing symptoms and signs depending upon how far along the disease process they present at. In secondary care, most patients present with symptoms and signs consistent with established disease, whereas in primary care patients may present at any stage of the disease. It is important that medical students and junior doctors appreciate the differing presentations of the same disease when they are seeing patients in primary care, as this may be something that they are not familiar with. Simple visual models can be helpful in this process, particularly when they are applied to symptom-based and case-based learning methods [2]. In Figure 1, the differing presentations of the same disease at points $\mathrm{A}, \mathrm{B}$ and $\mathrm{C}$ can be discussed, along with the difficulties that this creates when performing a single point in time clinical assessment at each of these points in time. A single point in time clinical assessment performed during the early and evolving stage of an illness may not elicit the clinical findings that are required to make a diagnosis, as they have yet to evolve, whereas a fully-evolved illness will have positive findings in the history, clinical examination and investigations, which enable a diagnosis to be made. For example, most clinicians will recognise the patterns of illness that exist for established appendicitis, or meningococcal disease but assessing a patient with a 6-hour history of non-specific, central abdominal pain, or a child with a short history of a fever is unlikely to yield anything that will enable the clinician to make a firm diagnosis. Worse than this, the lack of disease-specific findings for a "red line", serious illness at point A may lead to this diagnosis not even being considered, due to a lack of awareness that the early, non-specific findings might represent a serious illness in its early stage. The safe management of patients in primary care relies upon the appreciation that serious illnesses may present with symptoms and signs that are common to both serious and minor illnesses and that even the most well-performed clinical assessment will be unable to identify the patient who will subsequently become seriously ill. This is why risk assessment and safety-netting skills are fundamental to safe practice in primary care.

\section{Risk Assessment}

The World Health Organisation has identified that there is an urgent need to give patient safety considerations the highest priority

*Corresponding author: Paul Silverston MBChB, The Mill Barn, Mill Lane Exning, Suffolk CB8 7JW, United Kingdom, Tel: 01638 577729; E-mail: paul.silverston@btinternet.com

Received October 28, 2013; Accepted January 15, 2014; Published January 25 2014

Citation: Paul Silverston MBChB (2014) The Importance of Teaching Risk Assessment and Safety-Netting Skills in Primary Care. Primary Health Care 4: 146 doi:10.4172/2167-1079.1000146

Copyright: () 2014 Paul Silverston MBChB. This is an open-access article distributed under the terms of the Creative Commons Attribution License, which permits unrestricted use, distribution, and reproduction in any medium, provided the original author and source are credited. 
at all times and this also applies to reducing risk and improving patient safety during the consultation process [3]. Risk assessment during the consultation process involves determining whether or not there are any specific "red flag" risk factors present when performing a clinical assessment of the patient. The first part of that process is to recognise and truly appreciate the concept that illness is a dynamic process, such that there is a need to learn patterns of illness that take account of not only the classical presentations of illness when it is in its established form but also the symptoms and signs associated with that illness during its evolution. If medical students and junior doctors are not aware of these early, non-specific manifestations of serious illness, then they will not consider the potential for these illnesses being present and may overlook early and subtle "red flag" symptoms and signs during the clinical assessment. For example, a child with meningococcal disease may present initially with non-specific symptoms and signs, so its presence may not be considered when the child is first seen and examined. Whilst a diagnosis may not be possible at point $\mathrm{A}$ in Figure 1, when it will be indistinguishable from a minor illness, its presence should, nevertheless, still be considered. If the potential for its presence is not considered, then the need to safety-net the patient for its subsequent development will also not be considered.

In primary care, medical students and doctors need to learn patterns of illness that are both symptom-based and patient safety focused, so that there is an awareness of the "red flags" that are associated with the symptoms that patients present with. There are a number of books that can assist with acquiring the required "symptom-sorting" skills $[4,5]$. However, it should also be appreciated that the risks that need to be considered are not only those that are associated with obvious lifethreatening medical conditions. For example, a patient may develop an uncommon serious complication from what would normally be a minor illness, such as the local or systemic consequences of a streptococcal sore throat, or the patient may be locally or systemically vulnerable due to an underlying medical condition. A patient with diabetes or epilepsy who experiences gastroenteritis may not be at risk from that illness itself but from the inability to keep down diseasecontrolling medication. There may also be non-medical risks related to the presence of psycho-social factors, such as those that were identified during the Confidential Enquiries into Asthma Deaths in the Eastern Region of the UK [6,7]. This is why a risk assessment is important in avoiding some of the complications that may arise later, either as the disease evolves, or as a direct consequence of it.

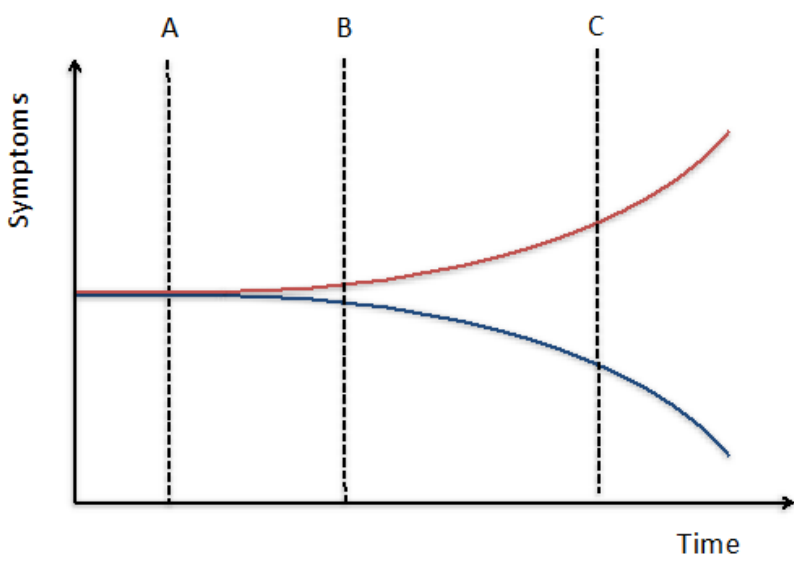

Figure 1: Symptoms vs. Time
A visual model can be used to encourage medical students and junior doctors to consider the risks that may be present at each step in the clinical assessment and to perform a risk assessment as they proceed through the assessment process [8]. This is best used with a symptom or case-based learning approach, so that the risks related to each symptom that the patient may present with can be risk assessed at each step of the clinical assessment process, from the presence of co-morbidities in the patient's medical history, to their medications, to the relevance of abnormal physiological findings in their clinical observations and physical findings in their clinical examination. This encourages a more holistic approach to risk assessment that simply considering whether the symptoms presented might be related to a life-threatening illness.

Whilst a risk assessment may enable the medical student or junior doctor to consider the possibility that the patient may have a potentially life-threatening illness, it may not be able to positively confirm or exclude it. The resulting diagnostic uncertainty needs to be managed safely. One of the ironies of medicine is that the very thing that creates the problem of diagnostic uncertainty, the relationship between illness and time, can also be used as a tool to aid diagnosis during the subsequent evolution of the illness. When diagnostic uncertainty arises, a number of options are available in primary care that utilise the passage of time and the progression of illness. It is important that medical students and junior doctors are introduced to the indications for each of these options, along with the clinical and sometimes non-clinical reasons (such as doctor, or patient/relatives anxiety levels) for selecting each option. One option is to admit the patient for a period of medical observation during which further re-assessments are performed by medical staff, until a point in time when it can be ascertained that the patient is no longer at risk of having an evolving serious illness. The second option is to arrange for the patient to be re-assessed again after a period of time determined by the clinician, at which point the natural history of any illnesses being considered would make it apparent that one was dealing with one illness or another. The third option is to send the patient home with specific safety-netting instructions for them to follow. Medical systems, financial, political and medico-legal considerations may all play a part in this decision-making process and it is important that these are discussed, as well as the relative merits and risks associated with each of these decisions.

\section{Safety-Netting Skills}

At the end of the clinical assessment phase of the consultation, a decision has to be made as to the management of the patient, which could include immediate admission for further medical observations, investigations, or treatment; urgent referral for investigations, or treatment; a planned review of the patient after a period of time; or sending the patient home with safety-netting advice. Admission for further observations, planned review and safety-netting all share a common denominator in that they rely upon the existence of the relationship between illness and time. In all three situations, it is expected that the progression of the illness over time will enable there to be a distinction between a serious illness that is becoming progressively worse and one that is improving over time. In other words, time is being used as a diagnostic tool. In patients who are being sent home with safety-netting advice, great care needs to be taken as to the appropriateness and quality of that advice and it is important that medical students and junior doctors learn the principles and practices of safety-netting.

A simple checklist can be used to introduce this subject, as a means of encouraging the medical student or junior doctor to consider the issues involved in safety-netting, known as the " $3 \mathrm{C}$ 's" checklist. The first 
"C" relates to an assessment of the Capability of the patient or relative to understand what is being discussed. This involves an assessment of mental capacity, on occasions mental competency, but always their intellectual and language skills to follow what can, at times, be quite complex medical concepts and psychomotor skills.

The next part of the process involves an assessment of the ability of the patient or relative to Comply with the instructions that are being given. The appropriateness of expecting a lay-person to re-assess the patient following a medical assessment should be considered, as well as their ability to comply with the instructions in practical terms. For example, when delivering instructions that involve checking the patient's temperature, pupillary reaction, or performing a blanch test for a rash, it should be confirmed that the equipment required to perform these tests is available to the patient or relative and that they know how to perform any tests required of them, however simple they seem. Psycho-social considerations may also have to be considered such as the ability of a parent to monitor a sick child at home when there are other children who are in need of attention; or if the child is shared between two parental homes, whether and how the instructions conveyed to one parent will be conveyed to the other.

Finally, an assessment of the patient's or relative's Comprehension is required to see that they have understood the instructions that have been given, both in terms of any tests that they are required to perform but also in terms of the implications of any positive findings and exactly what action should be taken. Ideally, written instructions should be provided as well as verbal instructions and the advice given should be documented in the patient's notes.

Whilst, safety-netting advice may be relatively straightforward when it applies to one condition that is being considered, such as the head injury advice given to patients who appear to have sustained a minor head injury, it can be much more complex when there are a number of differential diagnoses to consider. The principle aim of safety-netting is to ensure that patients re-present at point $\mathrm{B}$ in Figure 1, rather than at point $\mathrm{C}$, when it may be too late to avert a disaster. However, in the case of a child presenting with a short history of a febrile illness, the child might go on to develop a wide range of life-threatening illnesses, from meningococcal disease, to the complications of a streptococcal infection, to epiglottitis, to pneumonia, to urosepsis, to name but a few. Safety-netting patients for all these possibilities are not realistic. Thus, safety-netting advice must include a combination of both the diseasespecific symptoms and signs to look for, as well as how to identify a general deterioration in the patient's condition, along with permission to seek help again if there are any concerns. The criteria for seeking help again should be both specific (ie a non-blanching rash) and general (ie a change in the level of responsiveness) and the action that should be taken should be clear. In the UK, the introduction of symptom-based, patient safety focused guidelines such as the ones produced by the National Institute of Clinical Excellence for the assessment and management of feverish children and the guideline for assessing the need for antibiotic prescribing in patients with minor illness have emphasised the need for developing good safety-netting skills in primary care $[9,10]$.

\section{Summary}

Medical students and junior doctors involved in assessing patients within the primary care setting should appreciate the differing presentations and patterns of illness that exist within this clinical setting. The dynamic nature of illness and the limitations of a single point in time clinical assessment performed during the early and evolving stages of illness may lead to the presence of diagnostic uncertainty, which needs to be managed safely. Simple visual models can help medical educators explain the differences in illness presentations that arise depending upon the point in time that the patient presents, as well as the difficulties that arise in making a diagnosis, or excluding serious illness, when a patient presents during the early stages of a serious illness. The importance of performing a holistic risk assessment and the criteria used for deciding whether or not to admit a patient; review them later; or to send them home with safety-netting advice can also be discussed. This may also have the added benefit of helping to reduce some of the friction that sometimes arises at the primary/secondary care interface with regard to the appropriateness or otherwise of hospital referrals and admissions and concerns surrounding over and under-diagnosis of patients who present during the early stages of illness. Above all else, it encourages medical students and junior doctors to place patient safety at the heart of their consultations.

\section{References}

1. Silverston $P$, Stewart $L$ (2012) Teaching safe consulting. Student BMJ 20: e7791.

2. Silverston $P(2012)$ Using a model of illness to aid symptom-based learning in primary care. Educ Prim Care 23: 443-445.

3. Ellis O (2009) Putting safety on the curriculum. BMJ 339: b3725.

4. Hopcroft K, Forte V (2010) Symptom Sorter. Radcliffe Publishing Ltd; ISBN10:1846194539.

5. Sahib el-Rahidi (2011) Paediatric Symptom Sorter. Radcliffe Publishing Ltd; ISBN-10: 1846194741

6. Anagnostou K, Harrison B, lles R, Nasser S (2012) Risk factors for childhood asthma deaths from the UK Eastern Region Confidential Enquiry 2001-2006. Prim Care Respir J 21: 71-77.

7. Harrison B, Stephenson P, Mohan G, Nasser S (2005) An ongoing Confidential Enquiry into asthma deaths in the Eastern Region of the UK, 2001-2003. Prim Care Respir J 14: 303-313.

8. http://dx.doi.org/10.1016/j.nedt.2013.03.001

9. http://publications.nice.org.uk/feverish-illness-in-children-cg160

10. http:/publications.nice.org.uk/feverish-illness-in-children-cg69. 